\title{
Guía de práctica clínica: Diagnóstico y tratamiento de la cefalea en adolescentes y adultos: resumen de la guía NICE
}

Diagnosis and treatment of headaches in young people and adults (NICE Clinical Guideline 150)

Resumido y traducido con autorización del National Institute for Health and Clinical Excellence (NICE). Disponible en: http://www.nice.org.uk/nicemedia/live/13901/60853/60853.pdf

La cefalea constituye un problema de salud común que muchos médicos de atención primaria y secundaria encuentran difícil de abordar. Una vez excluidas las causas graves de cefalea (por ejemplo, infección, tumor, hemorragia y arteritis), la principal carga de enfermedad puede atribuirse a las denominadas cefaleas primarias (cefalea en racimos, migraña y cefalea tensional) y a las cefaleas por abuso de medicación. Este artículo resume las recomendaciones más recientes del Instituto Nacional para la Salud y la Excelencia Clínica (NICE) referidas al diagnóstico y tratamiento de las cefaleas en jóvenes y adultos.

\section{Recomendaciones}

Las recomendaciones del NICE se basan en revisiones sistemáticas de la mejor evidencia disponible, con consideraciones explicitas sobre costo-efectividad. Cuando la evidencia resulta escasa, las recomendaciones se basan en la experiencia del grupo de desarrollo de la guía (GDG) y en la opinión de lo que constituye una buena práctica. Los niveles de evidencia de las recomendaciones se expresan en cursiva y entre corchetes.

Evaluación: indicaciones para considerar la solicitud de estudios complementarios

- Evalúe a las personas que se presentan con cefalea y alguna de las siguientes características, y considere la necesidad de estudios complementarios o interconsulta (o ambos):

-Empeoramiento progresivo asociado a fiebre

-Cefalea de inicio súbito que alcanza su máxima intensidad a los cinco minutos

-Déficit neurológico de reciente inicio

-Deterioro cognitivo de reciente inicio

-Cambio en la personalidad

-Alteración del nivel de conciencia

-Traumatismo craneoencefálico reciente (por lo general dentro de los últimos tres meses)

-Cefalea provocada por tos, maniobras de valsalva, o estornudos -Cefalea provocada por el ejercicio

Tabla 1. Características diagnósticas de las cefaleas primarias.
-Cefalea ortostática (aquella que se modifica con los cambios posturales)

-Síntomas sugestivos de arteritis de células gigantes

-Síntomas y signos sugestivos de glaucoma agudo de ángulo cerrado

-Cambio sustancial en las características de la cefalea.

[Basados en la experiencia y opinión del GDG]

- Considere la necesidad de solicitar estudios complementarios o interconsulta (o ambos) en aquellas personas que se presentan con cefalea y aparición reciente de cualquiera de los siguientes:

-Inmunocompromiso, causado por ejemplo por VIH o fármacos inmunosupresores

-Edad menor a 20 años y antecedentes de cáncer

-Antecedentes de cáncer con posibilidad de metástasis en el cerebro

-Vómitos sin causa obvia.

[Basados en evidencia de muy baja calidad provenientes de dos estudios de cohortes, y de la experiencia y opinión del GDG]

\section{Diagnóstico}

- Establezca el diagnóstico de alguna de las cefaleas primarias considerando las características de la tabla 1.

[Basado en la experiencia y opinión del GDG, tomando los criterios de la Sociedad Internacional de Cefalea (ICHD-III)].

- Esté alerta a la posibilidad de cefalea por abuso de medicación en personas cuya cefalea comenzó o empeoró mientras se encontraban medicadas durante tres meses o más con algunos de los siguientes medicamentos:

-Triptanes, opioides, ergotamínicos (o su combinación), durante diez días al mes o más, o

-Paracetamol, aspirina, o anti-inflamatorios no esteroides (AINE), ya sea solos o en cualquier combinación, durante 15 días al mes o más.

[Basado en la experiencia y la opinión del GDG, tomando los criterios de la Sociedad Internacional de Cefalea (ICHD-II)].

\begin{tabular}{|c|c|c|c|c|c|c|}
\hline $\begin{array}{l}\text { Caracteristica } \\
\text { del dolor }\end{array}$ & \multicolumn{2}{|c|}{ Cefalea tensional } & \multicolumn{2}{|c|}{ Migraña (con o sin aura) } & \multicolumn{2}{|c|}{ Cefalea en racimos (tipo Cluster) } \\
\hline Localización? & \multicolumn{2}{|c|}{ Bilateral } & \multicolumn{2}{|c|}{ Unilateral o bilateral } & \multicolumn{2}{|c|}{$\begin{array}{l}\text { Unilateral (alrededor o sobre el ojo, } \\
\text { y al costado de la cara o cabeza) }\end{array}$} \\
\hline Calidad & \multicolumn{2}{|c|}{ Presión o tensión (no pulsátil) } & \multicolumn{2}{|c|}{$\begin{array}{l}\text { Pulsátil (terebrante } 0 \text { explosivo en } \\
\text { adolescentes de } 12 \text { a } 17 \text { años) }\end{array}$} & \multicolumn{2}{|c|}{$\begin{array}{l}\text { Variable (puede ser agudo, quemante, } \\
\text { terebrante, tensionante) }\end{array}$} \\
\hline Intensidad & \multicolumn{2}{|c|}{ Leve a moderado } & \multicolumn{2}{|c|}{ Moderado a severo } & \multicolumn{2}{|c|}{ Severo a muy severo } \\
\hline $\begin{array}{l}\text { Relación con la } \\
\text { actividad diaria }\end{array}$ & \multicolumn{2}{|c|}{$\begin{array}{l}\text { No agravado por las } \\
\text { actividades diarias }\end{array}$} & \multicolumn{2}{|c|}{$\begin{array}{c}\text { Agravado por, o hace evitar, las actividades } \\
\text { diarias }\end{array}$} & \multicolumn{2}{|c|}{ Inquietud 0 agitación } \\
\hline Otros síntomas & \multicolumn{2}{|c|}{ Ninguno } & \multicolumn{2}{|c|}{$\begin{array}{l}\text { Sensibilidad inusual a la luz o sonidos (0 } \\
\text { ambos), nausea o vómitos ( } 0 \text { ambos). Puede } \\
\text { ocurrir aura }{ }^{*} \text { con } 0 \text { sin la cefalea. }\end{array}$} & \multicolumn{2}{|c|}{$\begin{array}{l}\text { Del mismo lado de la cefalea: inyección conjuntival y/o } \\
\text { lagrimeo; congestión nasal y/o rinorrea; sudoración en } \\
\text { frente } 0 \text { cara; miosis y/o ptosis palpebral. }\end{array}$} \\
\hline Duración & \multicolumn{2}{|c|}{$\begin{array}{l}30 \text { minutos a síntomas } \\
\text { continuos }\end{array}$} & \multicolumn{2}{|c|}{$\begin{array}{c}4 \text { a } 72 \text { horas en adultos; } 1 \text { a } 72 \text { horas en } \\
\text { adolescentes de } 12 \text { a } 17 \text { años }\end{array}$} & \multicolumn{2}{|c|}{15 a 180 minutos } \\
\hline Frecuencia & $<15$ días al mes & $\begin{array}{l}\geq 15 \text { días al mes, } \\
\text { por mas de tres } \\
\text { meses }\end{array}$ & $<15$ días al mes & $\begin{array}{l}\geq 15 \text { días al mes, } \\
\text { por mas de tres } \\
\text { meses }\end{array}$ & $\begin{array}{l}\text { De uno día por medio a ocho } \\
\text { por día }{ }^{€} \text {, con remisiones } \\
\text { continuas }>1 \text { mes }^{\varepsilon}\end{array}$ & $\begin{array}{l}\text { De uno día por medio a ocho } \\
\text { por día }{ }^{€} \text {, con remisiones } \\
\text { continuas }<1 \text { mes }^{£}\end{array}$ \\
\hline Diagnóstico & $\begin{array}{l}\text { Cefalea tensional } \\
\text { episódica }\end{array}$ & $\begin{array}{l}\text { Cefalea tensional } \\
\text { crónica }\end{array}$ & Migraña episódica & Migraña crónica & Cefalea en racimos episódica & Cefalea en racimos crónica \\
\hline
\end{tabular}

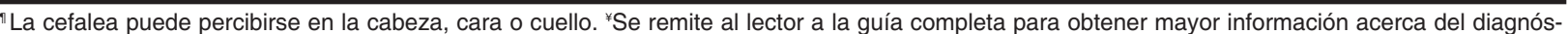
tico de migraña con aura. Los síntomas del aura son completamente reversibles, aparecen en aproximadamente cinco minutos y permanecen por cinco a 60 minutos. Las manifestaciones típicas incluyen: destellos luminosos, escotomas, pérdida parcial de la visión; entumecimiento o pinchazos cutáneos; y trastornos del habla. ${ }^{€}$ Frecuencia de episodios durante el brote. \&lntervalo libre de síntomas entre los brotes. 


\section{Manejo}

Para todas las cefaleas

- No solicite una neuroimagen en los pacientes con diagnóstico de cefalea tensional, migraña, cefalea en racimos y cefalea por abuso de medicamentos con el único objetivo de brindar reaseguro.

[Basado en evidencia de calidad muy baja a moderada de un ensayo controlado aleatorizado (ECA)]

- Al discutir el diagnóstico de cefalea con el paciente, contemple lo siguiente:

-Una explicación del diagnóstico efectuado y el reaseguro de que se han excluidos otros orígenes

-Las opciones de tratamiento

-El reconocimiento de que la cefalea es un trastorno médico concreto que puede tener un serio impacto en la persona, su familia y/o cuidadores.

[Basado en estudios observacionales con reportes cuya calidad oscilan de baja a buena]

- Explique el riesgo de cefalea por abuso de medicación a las personas que están utilizando tratamientos agudos para la cefalea.

[Basado en la experiencia y opinión del GDG]

Para la cefalea tensional

- Considere el uso de aspirina, paracetamol o un AINE para el tratamiento agudo, teniendo en cuenta las preferencias de la persona, sus comorbilidades y los riesgos de eventos adversos.

[Basado en la evidencia de baja calidad proveniente de ensayos controlados aleatorizados]

Debido a su asociación con el síndrome de Reye, no ofrezca preparados que contengan aspirina a individuos menores de 16 años.

- No ofrezca opioides para el tratamiento agudo.

[Basado en la ausencia de pruebas sobre efectividad y la experiencia y opinión del GDG]

- Considere la posibilidad de un curso de hasta diez sesiones de acupuntura durante cinco a ocho semanas para el tratamiento profiláctico de cefalea tensional crónica.

[Basado en evidencia de baja y muy baja calidad provenientes de ECA simple ciego]

\section{Para la migraña con o sin aura}

- Ofrezca tratamiento combinado con un triptan oral y un AINE, o un triptan oral y paracetamol para el tratamiento agudo, teniendo en cuenta las preferencias del paciente, las comorbilidades y el riesgo de eventos adversos. En los adolescentes de 12 a 17 años considere el uso de un triptan nasal en lugar de uno por vía oral.

[Basado evidencia de calidad muy baja a baja, proveniente de comparaciones directas de ECA meta-analizados en red* y su correspondiente análisis de costo efectividad*

- En los individuos en quienes las presentaciones orales (o presentaciones nasales en adolescentes) resulten ineficaces o no se toleren:

-Ofrezca el uso de metoclopramida o proclorperazina por vía parenteral, y

-Agregue un AINE o triptanos también por vía parenteral, si éstas no han sido ya utilizados.

[El primer punto se basa en evidencias de muy baja calidad proveniente de ECA. El segundo punto se basa en la experiencia y la opinión del GDG y en evidencia indirecta de ECA de baja a muy baja calidad]

- Ofrezca tratamiento preventivo con topiramato y propranolol de acuerdo a la preferencia de los pacientes, sus comorbilidades y el riesgo de eventos adversos. Informe a las mujeres y las adolescentes en edad fértil que el topiramato está asociado con un riesgo incrementado de malformaciones fetales y que este puede afectar además la eficacia de los anticonceptivos hormonales. Asegúrese ofrecer una adecuada anticoncepción. [Basado evidencia de calidad baja a alta, proveniente de comparaciones directas de ECA meta-analizados en red y su correspondiente análisis de costo efectividad]

- Si tanto topiramato como propranolol resultan inadecuados o ineficaces, considere un curso de hasta diez sesiones de acupuntura durante cinco a ocho semanas o gabapentina (hasta $1.200 \mathrm{mg} /$ día) de acuerdo a la preferencia del paciente, sus comorbilidades y riesgo de eventos adversos.

[Basado evidencia de calidad baja a alta, proveniente de comparaciones directas de ECA meta-analizados en red y su correspondiente análisis de costo efectividad]

- Asesore a las personas con migraña que la riboflavina (400 mg una vez al día) puede reducir la frecuencia y la intensidad de las migrañas en algunas personas.

[Basado en evidencia de calidad moderada proveniente de ECA]

Uso de anticonceptivos hormonales combinados en mujeres y adolescentes con migraña

- En pacientes que tienen migraña con aura, no ofrezca rutinariamente anticonceptivos hormonales combinados para la anticoncepción.

[Basado en la experiencia y la opinión del GDG]

\section{Migraña menstrual}

- Para las mujeres y las adolescentes con migraña desencadenada en el periodo menstrual que no responde adecuadamente al tratamiento agudo estándar, considere el tratamiento con frovatriptán (2,5 mg dos veces al día) o zolmitriptán (2,5 mg dos veces o tres veces al día) en los días en los que se esperan los episodios.

[Basado en evidencia de baja calidad proveniente de ECA y en la experiencia y opinión del GDG]

\section{Cefalea en racimos}

- Ofrezca oxígeno y un triptan por vía subcutánea o nasal para el tratamiento agudo.

[Basado en evidencia de calidad moderada proveniente de ECA]

- Cuando se utiliza el oxígeno para el tratamiento agudo:

-Utilice concentraciones al $100 \%$ a un flujo de al menos 12 litros por minuto con una máscara con reservorio, y organice la disponibilidad de oxígeno domiciliario y ambulatorio.

[El primer punto se basa en la evidencia de calidad moderada proveniente de ECA y de la experiencia y opinión del GDG. El segundo punto se basa en la experiencia y opinión del GDG]

- Cuando se utilicen triptanes por vía subcutánea o nasal, asegúrese de que la persona disponga a continuación de un adecuado suministro de tratamiento. Esto se debe calcular de acuerdo con la historia personal de episodios por ataque, teniendo en cuenta la dosis diaria máxima recomendada por el fabricante.

[Basado en la experiencia y la opinión del GDG]

- Considere el uso de verapamilo para el tratamiento profiláctico durante un ataque de cefalea en racimos. Si no está familiarizado con su uso para el tratamiento de la cefalea en racimos, interconsulte a un especialista antes de comenzar esta 
medicación, considerando además el requerimiento de monitoreo electrocardiográfico.

[Basado en evidencia de baja y muy baja calidad proveniente de un ECA]

\section{Cefaleas primarias durante el embarazo}

- Ofrezca a las mujeres embarazadas paracetamol para el tratamiento agudo de la migraña. Considere el uso de un triptan o un AINE después de discutir los beneficios y riesgos asociados con el uso de cada droga durante el embarazo.

[Basado en la evidencia de muy baja calidad proveniente de tres estudios de cohorte* y de la experiencia y la opinión del GDG]

- Interconsulte al especialista si se requiere tratamiento profiláctico de la migraña durante el embarazo.

[Basado en la experiencia y la opinión del GDG]

- Interconsulte al especialista si se requiere tratamiento para la cefalea en racimos durante el embarazo.

[Basado en evidencia de calidad baja y muy baja proveniente de un estudio de cohortes con población indirecta y la experiencia y opinión del GDG]

\section{Cefalea por abuso de medicación}

- Explique a los pacientes con cefalea por abuso de medicación que el tratamiento implica la retirada de los medicamentos que son usados en exceso.

[Basado en la evidencia de muy baja calidad proveniente de un ECA y de la experiencia y opinión del GDG]

- Aconseje a los pacientes a suspender los medicamentos usados en exceso durante al menos un mes, y a suspenderlos en forma abrupta en lugar de gradualmente.

[Basado en la experiencia y la opinión del GDG]

- Informe a los pacientes que los episodios de cefalea probablemente empeoraran en el corto plazo antes de mejorar y que pueden aparecer síntomas de abstinencia. Provea un seguimiento cercano para brindar apoyo según sea necesario. [Basado en la experiencia y la opinión del GDG]

- Considere la posibilidad de tratamiento profiláctico para la cefalea primaria subyacente, además de la suspensión de los fármacos utilizados en exceso.

[Basado en la experiencia y la opinión del GDG]

\section{Superando las barreras}

El uso de terapia combinada como tratamiento de elección para la migraña es innovador y debería mejorar el tratamiento agudo. El cumplimiento puede, sin embargo, ser mejor cuando las personas toman un único fármaco, alternativa que es ofrecida también en esta guía. Además de tener en cuenta otros puntos, la preferencia del paciente debe pesar al momento de elegir el tratamiento de la migraña aguda. En el momento de la publicación (septiembre de 2012), no todos los fármacos recomendados tenían autorización de comercialización en el Reino Unido para la indicación o indicaciones específicas así como para su uso en adolescentes. Así en estos casos, queda bajo la responsabilidad de los médicos la decisión de que fármaco prescribir siguiendo su criterio profesional. Debido a que el topiramato se recomienda como agente de primera línea para la profilaxis de la migraña, los médicos y los pacientes deben ser conscientes de su uso seguro en las mujeres y adolescentes en edad fértil. Su potencial efecto como inductor enzimático implica que el uso concurrente de anticonceptivos hormonales resulta poco fiable. Los médicos deberán consultar las guías locales (ej. British National Formulary, Royal College of Obtetricians and Gynaecologists Faculty of Sexual and Reproductive Healthcare) para aconsejar sobre el uso de anticonceptivos.

Tanto los médicos generales como las compañías de suministro de oxígeno deben garantizar su rápida provisión para el tratamiento de los pacientes con cefalea en racimo. Entre los retos relacionados con la cefalea por abuso de medicación se incluyen la necesidad de reconocer sus factores de riesgo y brindar un temprano asesoramiento preventivo. En estos casos la recomendación de suspender abruptamente la medicación vigente puede no ser bienvenida, especialmente si se tiene en cuenta que el diagnóstico definitivo sólo puede hacerse después de resuelta la cefalea, cosa que sólo ocurre en la mitad de los pacientes que logran suspender el uso de su medicación habitual.

Debido a la ausencia de evidencia, no se han establecido recomendaciones referentes a otras intervenciones como la terapia manual, ejercicio, terapia cognitivo-conductual, o programas de autogestión. La guía hace recomendaciones de investigación en algunas de estas áreas.

Fuente de financiamiento: National Institute for Health and Clinical Excellence (NICE).

Nota: las guías NICE son desarrolladas para su aplicación dentro del Servicio Nacional de Salud (sigla en ingles NHS) en el Reino Unido. EI NICE no toma responsabilidad alguna por la traducción al español aquí publicada de la presente guía.

Resumido de: National Institute for Health and Clinical Excellence. Headaches. Diagnosis and management of headaches in young people and adults. (Clinical Guideline 150.) 2012. Disponible en URL:http://guidance.nice.org.uk/CG150/Guidance (ultimo acceso: setiembre 2012). Evid Act Práct Ambul. Jul-Set 2012;15(3):112-114. 